\title{
Infrared Spectra of Young Stars Embedded in the R Coronae Australis Cloud
}

\author{
Michael R. Meyer \\ Steward Observatory, The University of Arizona, Tucson, AZ 85721; mmeyer@as.arizona.edu \\ AND \\ BRUCE A. WILKING \\ Department of Physics and Astronomy, University of Missouri-St. Louis, St. Louis, MO 63121; bwilking@umsl.edu \\ Received 2008 December 1; accepted 2009 February 27; published 2009 May 14
}

\begin{abstract}
Infrared spectra are presented for a magnitude-limited $(K<12.5 \mathrm{mag})$ sample of stellar objects observed toward the R Coronae Australis molecular core. These spectra, which are used to identify young stellar objects in the cloud, include the wavelengths of emission lines from [Fe II] and $H_{2}$, four of the Brackett series lines, the $\mathrm{CO}$ bandheads, as well as photospheric absorption lines of $\mathrm{Al}, \mathrm{Na}, \mathrm{Mg}, \mathrm{Si}$, and $\mathrm{Ca}$. For a subset of the sample, the spectra are compared to those of infrared spectral standard stars to derive spectral types and luminosity classes. By comparing their placement in a Hertzsprung-Russell diagram with theoretical pre-main-sequence tracks and isochrones, we estimate the stellar masses and assess the evolutionary states of the members of this young aggregate. All of the sources classified via near-IR spectroscopy have masses in the range $0.2-2.5 M_{\odot}$. The locus of points in the H-R diagram is lower than observed for other embedded clusters (e.g., NGC 2024 and the $\rho$ Oph core), suggesting either a more advanced evolutionary state or a difference in the intrinsic stellar birthline for very young clusters. We discuss the implications of our results for the shape of the initial mass function of the embedded young cluster and the star-forming history of the cloud.
\end{abstract}

\section{INTRODUCTION}

It is thought that most stars in the disk of the Milky Way form in embedded young clusters, which evolve into unbound associations on timescales of $10 \mathrm{Myr}$ or less. As a result, measuring the star-forming histories and initial mass functions of these regions provides fundamental constraints to theories of star formation (Lada \& Lada 2003). For example, molecular clouds could survive for $>10 \mathrm{Myr}$ if they evolve from a magnetically-supported quasi-hydrostatic state into magnetically supercritical cores through the process of ambipolar diffusion. In this case, one might expect a range of stellar ages for stars embedded within the cloud depending on the details of the local field and cloud density (Shu et al. 1987). Alternatively, molecular clouds could be short-lived entities $(<10 \mathrm{Myr})$ that are rapidly created and destroyed through the combined effects of supernovae, winds from massive stars, and outflows from young stars (Palla \& Stahler 2000; Hartmann 2001). In this instance, we might expect to see a narrow range of stellar ages within an existing cloud, or even smooth age gradients in stellar populations observed across a region of space that recently hosted a giant molecular cloud.

The R Coronae Australis molecular cloud core contains the young intermediate mass star for which it is named, as well as dozens of lower luminosity sources (see Neuhäuser \& Forbrich 2008 and references therein). Ringing the star R $\mathrm{CrA}$ is an embedded cluster of about 10 young stellar objects referred to as the "Coronet" (Taylor \& Storey 1984, hereafter TS84). In addition to its proximity ( $\sim 130 \mathrm{pc}$; de Zeeuw et al. 1999; Marraco \& Rydgren 1981), it is an interesting target for further study for several reasons. The R CrA cloud core hosts an intermediate density aggregate with properties in between the rich Orion Trapezium cluster and sparse $T$ associations such as that found in the Taurus-Auriga dark cloud. Such aggregates may be a precursor of loose associations such as the TW Hya and $\eta$ Cha groups, whose members are in an important evolutionary phase with respect to planet formation and stellar angular momentum evolution. By investigating the $\mathrm{R} \mathrm{CrA}$ embedded population, we might learn about the "prehistory" of these groups and whether what we infer about early stellar evolution or planet formation is the exception or the rule in galactic disk star formation. Finally, its lack of obvious association with the Gould's Belt makes R CrA somewhat unique among dark clouds within $150 \mathrm{pc}$ and may tell us something important about the star-formation history of the solar neighborhood.

While previous infrared photometric surveys have uncovered a number of apparently embedded objects, spectroscopic studies have focused on the optically-visible T Tauri stars (Marraco \& Rydgren 1981; Walter et al. 1997) and widely distributed X-ray sources (Neuhäuser et al. 2000). In order to understand fully the star-forming history of the entire R CrA cloud as well as constrain the shape of the initial mass function of young stars, the young embedded population needs to be fully characterized. 
Developments in infrared instrumentation as well as techniques for analyzing these data have made it possible to locate embedded populations such as the R CrA cluster in the Hertzsprung-Russell (H-R) diagram and compare the positions of the sources with theoretical models of pre-main sequence (PMS) evolution as a function of mass. Here we describe the results of our near-infrared spectroscopic survey, aimed at determining the masses and ages of the embedded stellar population. In $\S 2$, we describe the observations and data reduction, while in $\S 3$ we describe the results derived from our spectroscopic survey. In $\S 4$ we discuss the implications of these results for star-formation in the region, and in $\S 5$ we summarize our main conclusions.

\section{OBSERVATIONS AND DATA REDUCTION}

Candidate cluster members were chosen from the IR photometric survey of Wilking et al. (1997, hereafter WMB97) on the basis of their position in the $M_{H}$ vs. $(J-H)$ color-magnitude diagram as well as the $(J-H)$ vs. $(H-K)$ color-color diagram. In Figure 1, we plot the sources for which we obtained near-infrared spectra. The brighter sources are either more massive or possess larger IR excess emission, while the observed colors of the sources reflect a combination of intrinsic wavelength-dependent photospheric flux ratios, differential extinction, and IR excess emission. Sources were chosen to be representative of the photometric sample, spanning a range of reddenings $\left(A_{V}=0-40\right)$, intrinsic luminosity $\left(0<M_{H}<\right.$ $6)$, and infrared excess emission $(0<\Delta K<0.8$; see $\S 4.1)$. The faintest stars observed as part of this program were $K \sim 12.5^{m}$.

The spectra were obtained with CTIO InfraRed Spectrometer (IRS, see DePoy et al. 1990; upgraded with a $256 \times 256 \mathrm{InSb}$ detector by R. Elston in 1994) on the Blanco $4.0 \mathrm{~m}$ telescope on 1995 June $9-11$ and 1996 June 4-7. We used the $751 \mathrm{~mm}^{-1}$ grating in second order for the $K$-band and third order for the $H$-band providing a mean resolving power of 1650 and 1800, respectively. A single grating position centered at $1.67 \mu \mathrm{m}$ was observed in the $H$-band and two positions centered at 2.19 and $2.30 \mu \mathrm{m}$ were observed in the $K$-band. Stellar spectra were obtained by nodding the source along the slit with a separation of $10^{\prime \prime}$ in an $A-B-B-A$ beam-switching mode. Telluric standards were observed at a variety of airmasses at the same grating settings as our program stars. Spectral standards were also observed in addition to embedded cluster members in order to derive spectral types.

The data were reduced using Image Reduction and Analysis Facility (IRAF) ${ }^{1}$. After applying a linearity correction to all images, beam-switched image pairs were subtracted from each other (e.g., $A-B$ and $B-A$ ) and corrected for pixel-to-pixel variations in sensitivity using two-dimensional flat fields. The

\footnotetext{
${ }^{1}$ IRAF is distributed by NOAO for AURA, Inc.
}

flat fields were constructed for each grating by using the difference between images of a target screen observed with and without illumination and normalized to 1.0. One-dimensional spectra were extracted from the two-dimensional calibrated images using the APALL package from the ONEDSPEC module of IRAF. The spectral trace was followed across the image using a cubic-spline function. Nearby residual sky spectra were also extracted and subtracted from the target spectra after linear interpolation along the slit providing a "second-order" background correction, important when the sky background varied between exposures $A$ and $B$ of a beam-switched sequence as well as removing any nearby extended emission-line nebulosity.

Wavelength calibration was established for each slit position using $\mathrm{OH}$ lines from the night sky or helium-argon emission lines from arc lamps. For the $K$-band spectra only, absorption from the Earth's atmosphere was corrected by dividing each extracted spectrum by a standard star (usually the A0 star HD 176386 in the R CrA cloud) whose hydrogen absorption lines in the stellar photospheric absorption spectrum were removed after performing Gaussian fits to the line profiles. Before division, we cross-correlated the individual target spectra with the telluric standard over the wavelength range most affected by telluric absorption to make sure there were no residual shifts in the wavelength solution between the standard and the program stars. No attempt was made to restore the true continuum shape of the final spectra; this could be approximated by multiplying each spectrum by a blackbody corresponding to the effective temperature of the telluric standard. Individual relative flux-calibrated spectra were then averaged together and divided by the median flux to create the final spectra shown in Figure 2. We estimated the signal-to-noise ratio (S/N) from the RMS measured in regions free from spectral lines or strong telluric absorptions from the final reduced spectra. For bright targets, such as those shown in Figures 2 and 3 ( $H<12$ and $K<$ 10.5), S/N varies from 30-100. For fainter targets $(14<H<12$ and $12<K<10.5), \mathrm{S} / \mathrm{N}$ varies between $10-30$.

\section{SPECTRAL ANALYSIS}

\subsection{Description of the Spectra}

Several interesting features are immediately apparent in the reduced data. Four stars exhibit $\mathrm{Br} \gamma$ in emission: [TS84] IRS 2, HH 100-IR, R CrA, and HA 2. Of these, only IRS 2 and HH 100-IR show signs of obvious Brackett emission in the $H$-band. HH 100-IR is unique in our sample as the only object which shows first-overtone $\mathrm{CO}$ emission in the $K$-band. ${ }^{2}$ IRS 2, $\mathrm{HH}$ 100-IR, and $\mathrm{R} \mathrm{CrA} \mathrm{distinguish} \mathrm{themselves} \mathrm{within} \mathrm{our}$

\footnotetext{
${ }^{2}$ Such emission has been successfully modeled as hot circumstellar gas in Keplerian rotation about a central protostellar object in sources such as WL 16 (Carr et al. 1993).
} 

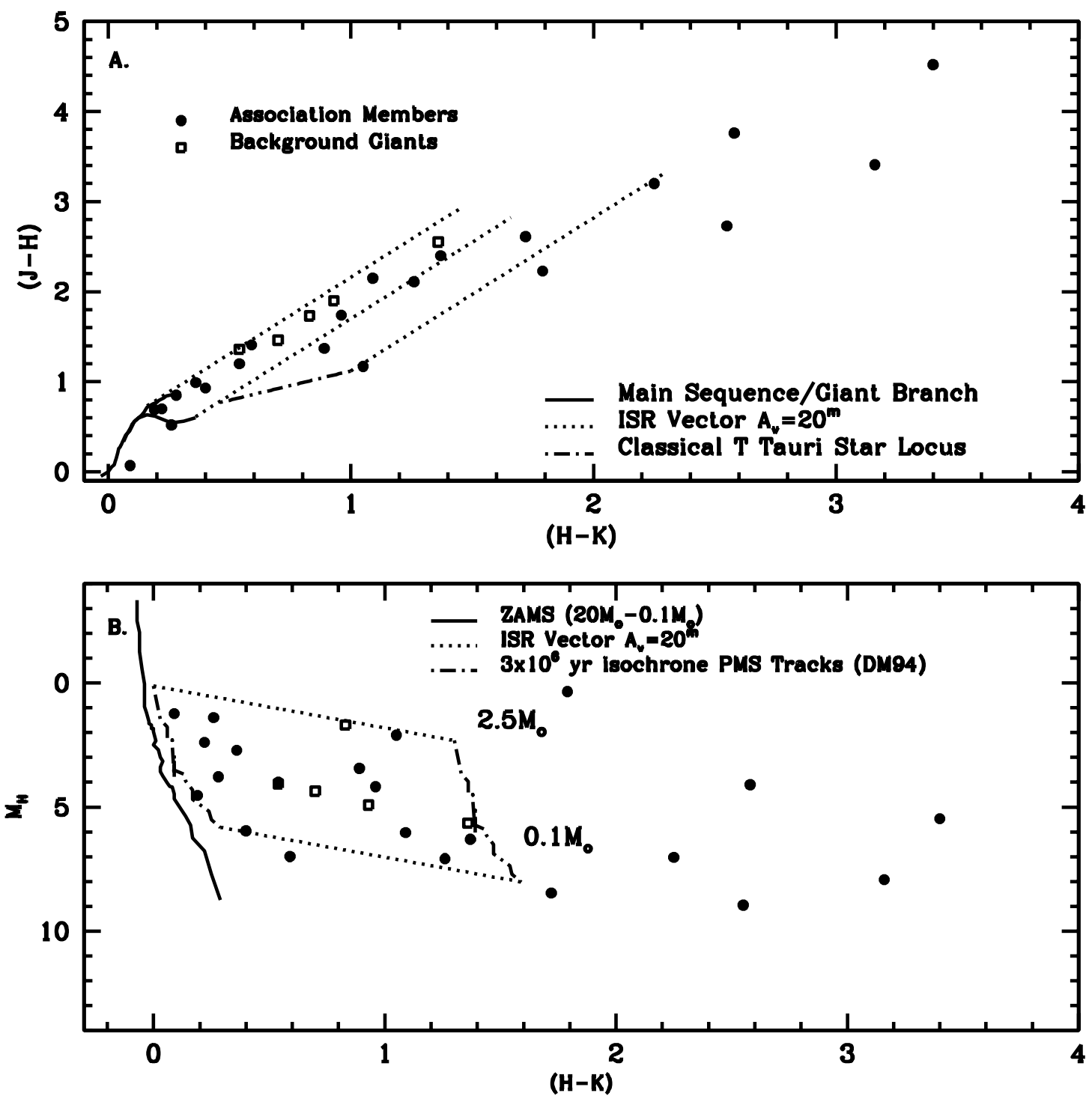

FIG. 1.-Color-color $(A)$ and color-magnitude $(B)$ diagrams shown for association members with data taken from Wilking et al. (1997). In the color-color diagram, we mark the normal colors of dwarf and giant stars, as well as the interstellar reddening vector from Cohen et al. (1981) transformed into the CIT system, and the classical T Tauri locus from Meyer et al. (1997). In the color-magnitude diagram, we show the zero-age main sequence as well as an isochrone transformed from D'Antona \& Mazzitelli (1994), along with the interstellar reddening vector from Cohen et al. (1981).

sample not only in their emission-line properties, but also as some of the most luminous objects in our sample lacking detectable photospheric features. Most of the spectra in our sample exhibit photospheric absorption features typical of intermediate and late-type stars. None of the objects have helium lines in absorption, characteristic of early-type OB stars. None of the objects in our sample show emission lines due to [Fe II] or $\mathrm{H}_{2}$, commonly observed in Class I young stellar objects (e.g., Greene \& Lada 1996). We also note that a spectrum obtained for S CrA (not formally within the Coronet cluster) reveals that it is a continuum + emission-line source exhibiting Brackett emission lines in the $K$ - and $H$-bands.

\subsection{Deriving Spectral Types}

We used the combined spectral information in the $H$ - and $K$-bands in order to derive spectral types for the stars in our sample. Spectral types were adopted through visual comparison with a grid of spectral standards obtained with the IRS and supplemented by standards reported in Meyer (1996), Meyer et al (1998), and Wallace \& Hinkle (1997). In the $H$-band, we used diagnostic atomic features such as the Al I triplet (1.67$1.68 \mu \mathrm{m}), \mathrm{Mg} \mathrm{I}(1.71 \mu \mathrm{m}), \mathrm{Ca} \mathrm{I}(1.616 \mu \mathrm{m}), \mathrm{Fe} \mathrm{I}(1.58 \mu \mathrm{m})$, and the Brackett lines of hydrogen (4-11, 4-12, 4-13). Other molecular features used in the analysis were $\mathrm{CO}(6,3)$ 


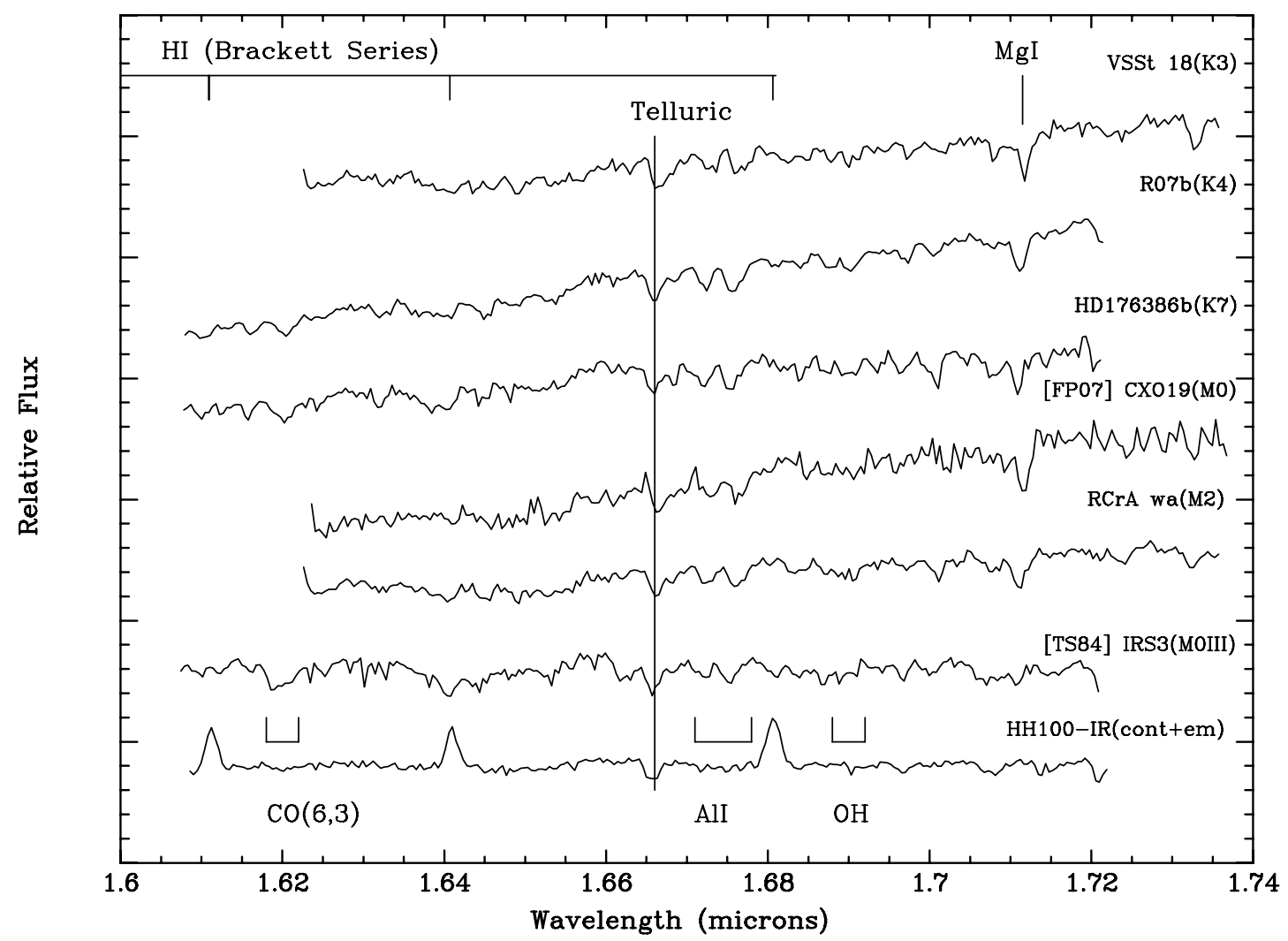

FIG. 2.- $H$-band spectra obtained with the CTIO IRS. Late-type features due to second-overtone CO absorption at $1.62 \mu \mathrm{m}$ and $\mathrm{Al} \mathrm{I}$ at $1.67 \mu \mathrm{m}$ are visible in the latetype stars, as well as features of $\mathrm{Mg}$ I and $\mathrm{Ca}$ I in the $F-G-K$ stars. Early-type stars show the Balmer $\mathrm{HI}$ lines in absorption while the deeply embedded Class I source HH100-IR shows hydrogen emission-lines.

$(1.619 \mu \mathrm{m})$ and $\mathrm{OH}(1.689 \mu \mathrm{m})$. Given the $\mathrm{S} / \mathrm{N}$ of the spectra, the presence of the Brackett lines in absorption indicated a star of spectral type $<\mathrm{G} 0$. However, these lines can be filled in by emission so that the absence of Brackett absorption lines does not rule out a spectral type $<\mathrm{G} 0$ for the star in question. Metallic lines of Fe I, Si I, and Mg I dominate the spectra of $G$ and early $K$ stars, while $\mathrm{Al} \mathrm{I}, \mathrm{Ca} \mathrm{I}, \mathrm{CO}$, and $\mathrm{OH}$ are stronger in the spectra of late-type stars K4-M5 (see Meyer et al. 1998 for a detailed analysis of the $H$-band spectra of MK spectral standards). The strength of the $\mathrm{CO}$ feature at $1.619 \mu \mathrm{m}$ can also provide some information concerning the surface gravity of the star. Those with giant-like surface gravities are easily distinguished from their dwarf star counterparts.

In the $K$-band we relied upon atomic features of the $\mathrm{Na} I$ doublets $(2.21 \mu \mathrm{m}$ and $2.34 \mu \mathrm{m})$, the Ca I triplet $(2.26 \mu \mathrm{m})$, $\operatorname{Mg~I}(2.28 \mu \mathrm{m})$, and $\operatorname{Br} \gamma(2.166 \mu \mathrm{m})$. As many as four molecular bands of $\mathrm{CO}$ are observed in some of our spectra (2-0, $3-1,4-2$, and 5-3). While the $K$-band $\mathrm{CO}$ features are stronger in giants than dwarfs just like their counterparts in the $H$-band, the $\mathrm{Na}$ I features are weaker in the giants. Again, the presence of $\mathrm{Br} \gamma$ in absorption confirms an early-type spectrum while absorption features of $\mathrm{Ca} \mathrm{I}$ and $\mathrm{Mg}$ I dominate intermediate spec- tral types. The spectra of late-type stars are dominated by $\mathrm{CO}$ and $\mathrm{Na}$ I absorption features.

Our derived spectral types are given in Table 1 and are averages of those determined from the $H$ - and $K$-band spectra. VSSt 18 (early $K$ ) and R CrA wa (early $M$ ) are high-S/N examples of their spectral types that lack IR excess. In a few cases, no absorption lines were seen and the the spectra were designated "continuum plus emission-line" objects. Some stars appear to have spectral types earlier than G0, indicated by the presence of Brackett absorption lines. These stars are particularly difficult to classify in the near-IR and thus have large errors (TY CrA and T CrA). Fortunately, these objects have been observed in the visual where early-type stars are best classified. In most cases, spectral types derived from the $K$-band agreed with those derived in the $H$-band and unambiguous designations were adopted with uncertainties typically \pm 2 subclasses for stars G0-M5. Because the strength of several features are double-valued with a maximum between K5-M0, occasionally we are unable to distinguish between spectral type K1-3 and M2-4. In three such cases ([GP75] R CrA wa, VSSt 13, and HA 2) we were able to break the degeneracy in our derived spectral types through comparison with visual spectra from other work. These spectral types are listed in Table 1 along with 


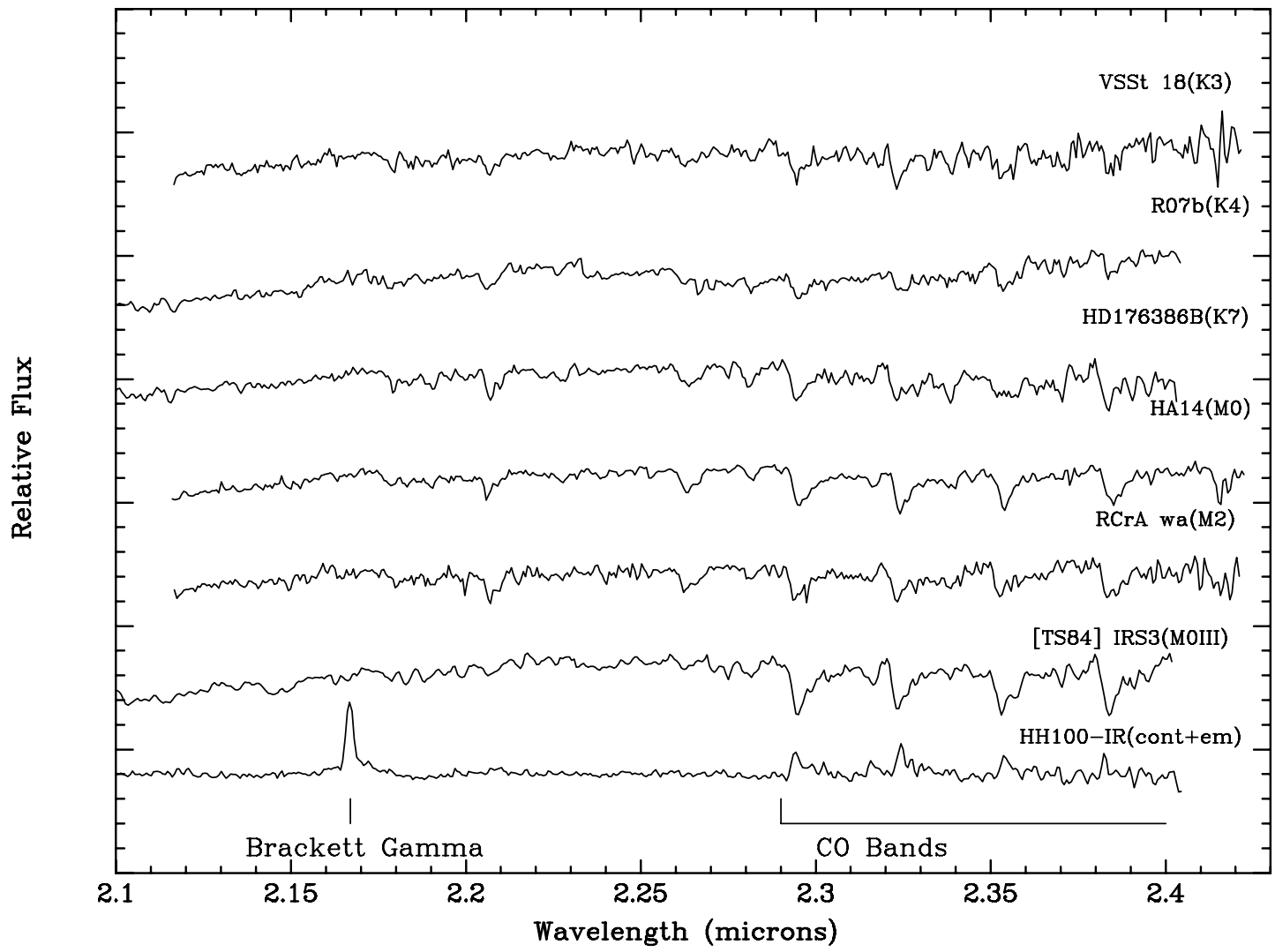

FIG. 3.- $K$-band spectra obtained with the CTIO IRS. Late-type features due to first-overtone CO absorption at $2.3 \mu \mathrm{m}$ and Na I $2.2 \mu \mathrm{m}$ are visible in the late-type

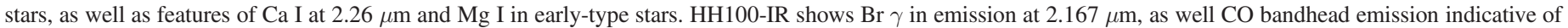
heavy accretion.

the IRS-derived spectral types. For five of the stars in our sample, we were only able to determine that the objects were "late-type," i.e., with spectral types $>$ K2-K4 ([TS84] IRS 13, [WMB97] 185848.1-365808, [GP75] R CrA wb, [TS84] IRS 15, and [TS84] IRS 8). Five stars in our sample turned out to be background giant stars based on the strength of the $\mathrm{CO}$ or $\mathrm{Na}$ I bands in their spectra compared to the strength of the other atomic features ([WMB97] 185800.6-370056, [TS84] IRS 3, [TS84] IRS 4, [WMB97] 185903.8-371005, and VSSt 7). For IRS 13 and [TS84] IRS 5, we applied the analysis of Greene \& Meyer (1995) to the spectra published in Greene \& Lada (1996) in order to derive the spectral types listed in Table 1. Finally, we obtained a low-resolution grism spectra of [TS84] IRS 9 with NSFCAM at the IRTF. Based on the analysis described in Wilking et al. (1999), we derived a spectral type based on the water bands. Two of the sources were unclassifable ([WMB97] 185815.3-370435 and [TS84] IRS 14) due to low $\mathrm{S} / \mathrm{N}$ and are not presented in Table 1. As shown in column (7) of Table 1, we find good agreement with spectral types previously published for late-type stars by Patten (1998) and Nisini et al. (2005).

\section{DERIVED SOURCE PROPERTIES}

\subsection{The H-R Diagram}

Whenever possible, we adopted extinction as calculated from the optical $(R-I)$ color index and applied bolometric corrections to dereddened $I$-band photometry in order to estimate the stellar luminosity. However, in many cases we were forced to estimate the extinction from near-infrared photometry alone as well as to estimate stellar luminosities from bolometric corrections applied to dereddened $J$-band data. We followed the procedures outlined in Meyer (1996) to calculate the amount of selective extinction suffered by our target stars based on near-infrared colors, using a standard reddening vector in the CIT system (Cohen et al. 1981). ${ }^{3}$ For stars with obvious IR excess in the $(J-H)$ vs. $(H-K)$ diagram, we dereddened them until they intersected the locus of intrinsic colors occupied by classical T Tauri stars found in the Taurus dark cloud (Meyer

\footnotetext{
${ }^{3}$ This reddening vector is consistent with the position of the five background giants (noted in Table 1) in the color-color diagram (Fig. 1), though formally different than that derived in Wilking et al. (1997).
} 


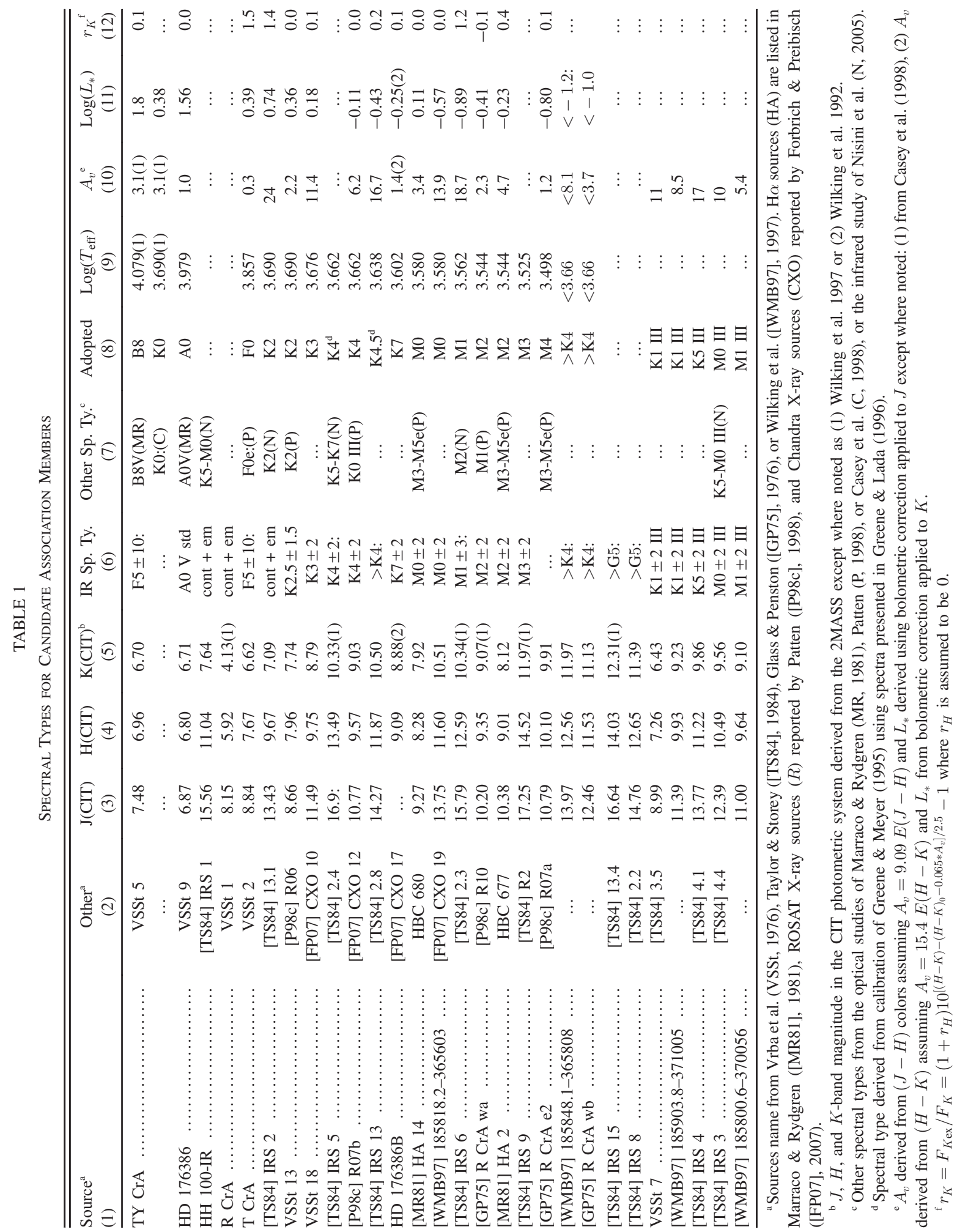




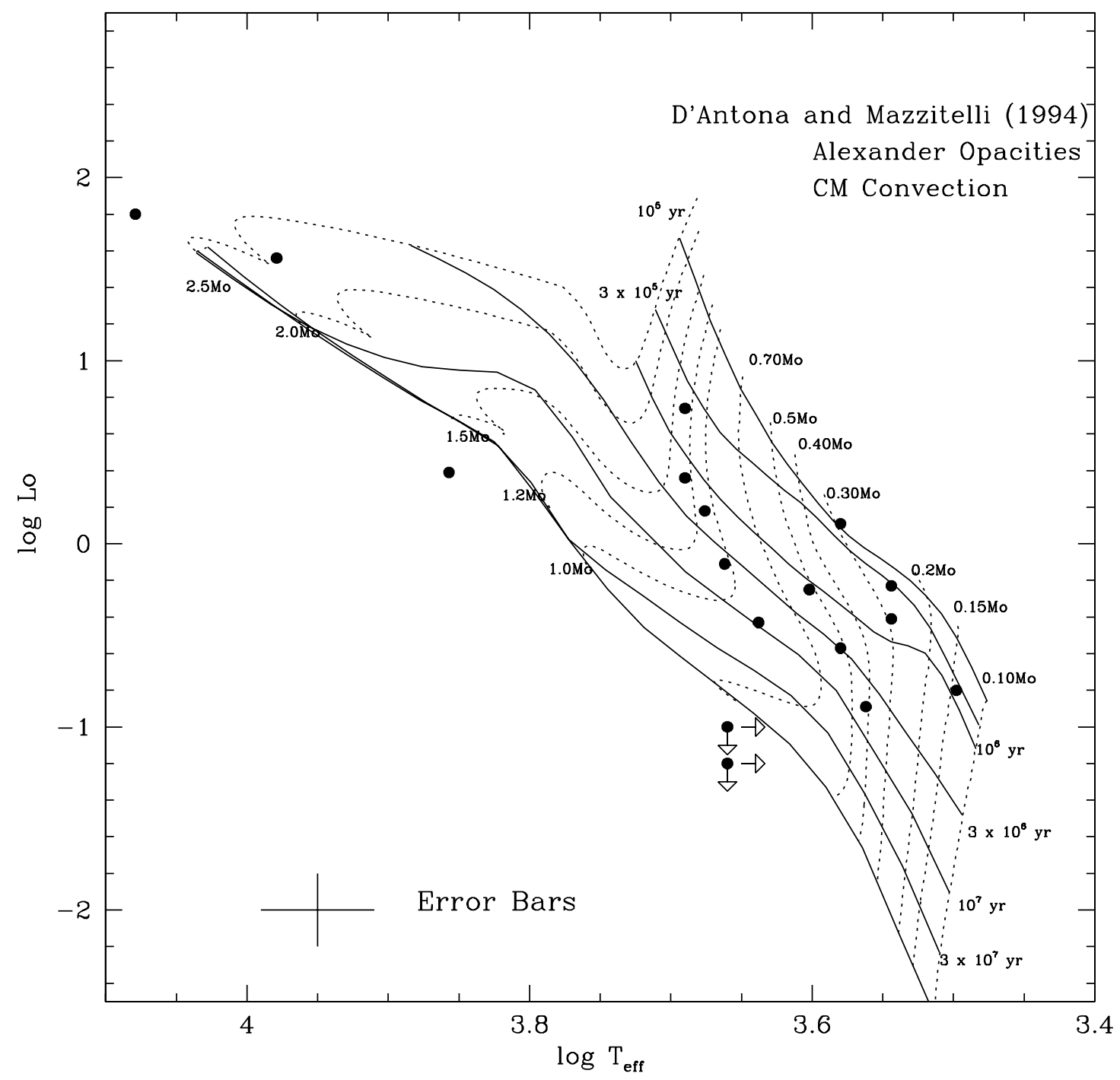

FIG. 4.-H-R diagram for embedded sources in R CrA plotted in comparison to the PMS tracks of D'Antona \& Mazzitelli (1994). The two stars with upper limits in $T_{\text {eff }}$ and luminosity are 185848.1-365808 and R CrA wb.

et al. 1997). For stars which lie within the reddened main sequence in the $J H K$ color-color diagram, we dereddened them in the $(J-H)$ versus $M_{J}$ color-magnitude diagram to a "mean isochrone" corresponding to the PMS models of D'Antona \& Mazzitelli (1994). Exceptions to this procedure include TY CrA (for which the detailed spectroscopic analysis of Casey et al. 1998 was adopted for this spectroscopic multiple system) and HD 176386B (for which only $H$ - and $K$-band data were available), as well as [WMB97] 185848.1-365808 and R CrA wb, where we estimated an upper limit to the extinction (and luminosity) assuming the earliest spectral type consistent with our analysis. The results of these calculations are reported in Table 1 where the adopted extinctions and stellar luminosities are listed.

In order to translate spectral types to effective temperature, we again follow the prescription of Meyer (1996). For stars earlier than K5, the Schmidt-Kaler (1982) conversion was adopted, and for later type stars, we used Bessell (1991). The results are given in Table 1. Using these data we construct the H-R diagram presented in Figure 4. Comparing the positions of our stars to the evolutionary models of D'Antona \& Mazzitelli (1994), we estimate the masses and ages of our target stars. The masses inferred for our sample range from $0.2-1.2 M_{\odot}$. The apparent ages derived from our H-R diagram range from $3 \times$ $10^{5}$ to $3 \times 10^{7} \mathrm{yr}$. However, the colors of some stars may be dominated by scattered light, and thus appear too blue for the temperature and true extinction toward the PMS star. Such arguments are occasionally made to explain the unusual position of some PMS stars in H-R diagrams, and in a few cases can be borne out through high-resolution imaging and mid-IR spectroscopy (e.g., Luhman et al. 2007). While this phenomenon 
might explain the apparent discrepancy between the low position in the H-R diagram and the evolutionary state of a few sources (judging by the shape of their spectral energy distributions), it is possible that there is an intrinsic age spread in the region.

\subsection{Circumstellar Properties}

Using the procedure described in $\S 4.1$, we can estimate the expected absolute $K$-band magnitude for our target sources. With the observed $(H-K)$ color, the extinction estimate described above, the expected $(H-K)$ intrinsic color associated with the known stellar spectral type, and assuming that each does not exhibit excess emission in the $J$ - or $H$-bands, we can estimate a lower limit to the $K$-band excess as follows:

$$
\Delta K=E(H-K)_{o}=(H-K)-(H-K)_{o}-A_{V} * 0.065,
$$

where we have adopted the reddening law of Cohen et al. (1981). Further, we can calculate the ratio of stellar emission to excess emission in the $K$-band following Meyer et al. (1997),

$$
\Delta K=E(H-K)_{o}=-2.5 \log \left[\left(1+r_{H}\right) /\left(1+r_{K}\right)\right],
$$

where we assume that $r_{H}=0$ and calculate a lower limit on the veiling parameter $r_{K}=r_{K \mathrm{ex}} / r_{*}$. These values are listed in Table 1 for all sources for which we were able to derive a spectral type and extinction estimate. These values are consistent with those typical for $\mathrm{T}$ Tauri stars in the Taurus dark cloud (Meyer et al. 1997). The largest excesses were estimated for T CrA, IRS 6, and HA 2. The quoted values assume that the spectral types and extinction estimates are accurate. Future work measuring the spectroscopic veiling should be undertaken to check these preliminary estimates (e.g., Doppmann et al. 2005).

\section{THE STELLAR AGGREGATE ASSOCIATED WITH R CrA}

We can use information from the H-R diagram to better constrain the properties of the $\mathrm{R} \mathrm{CrA} \mathrm{embedded} \mathrm{cluster.} \mathrm{This}$ enables us to construct an extinction-limited sample of stars from the photometric survey of Wilking et al. (1997) to study the shape of the IMF and the fraction of stars with circumstellar disks. Following Meyer (1996; see also Carpenter et al. 1997; Wilking et al. 2004) we construct an extinction-limited sample for all stars within $A_{V}<20^{m}$, which corresponds to the flux limit of our sample for a 3 Myr-old star at the hydrogen burning limit. For this sample of 45 stars, the ratio of high- to low-mass stars is $0.15 \pm 0.06$, consistent with having been drawn from the field star IMF (Meyer et al. 2000). Furthermore, the fraction of stars exhibiting a near-IR excess in the $J H K$ color-color diagram, within this extinction-limited photometric subsample, is $0.67 \pm 0.12$, consistent with other young clusters of similiar age (Haisch et al. 2001). This fraction is higher than that derived in Wilking et al. (1997), in part because our sample is $A_{V}$-limited (fewer background stars), and in part because we adopted a steeper slope for the reddening vector. We note that we have not considered the wider star formation environment that might include nearby early-type stars HR 6970 and HR 6971. Of note also is the faint companion to R CrA wa (dubbed R CrA wb) that could be a brown dwarf. Further work utilizing deeper infrared imagery combined with data from the Spitzer Space Telescope are needed to probe the substellar IMF and disk properties of the cluster (Sicilia-Aguilar et al. 2008).

There is some hint of a modest age spread in the R CrA cloud from 0.3-3 Myr consistent with those found in other clusters (Mamajek et al. 2002). However, given the error bars in our H-R diagram (cf. Hartmann 2001), we can only say that star formation is consistent with a spread of $\delta \tau<3 \mathrm{Myr}$. This imples that the typical radius for stars in this $\mathrm{H}-\mathrm{R}$ diagram is $1.5 R_{\odot}$, compared to $3.3 R_{\odot}$ in Ophiuchus and $3.6 R_{\odot}$ in NGC 2024. However, the observed stellar density $\left(\sim 1000 \mathrm{star}^{-3}\right)$ and the 1:1 ratio of Class I to Class II spectral energy distributions observed in the cluster imply that the cluster is probably younger. Assuming that all stars formed in such dense aggregates are intrinsically very young, intercomparison of these H-R diagrams could be interpreted as differences in intrinsic stellar birthlines (e.g., Stahler 1988).

What is the global star-forming history of the R CrA cloud? The young X-ray sources located to the west of R CrA appear to be significantly older than the sources found in the $\mathrm{R} \mathrm{CrA} \mathrm{core}$ (Neuhäuser et al. 2000, their Figure 1). Using the same methodology described in $\S 4.1$, we have placed these stars in the $\mathrm{H}-\mathrm{R}$ diagram and estimate ages from 3-10 Myr. Other young stars in the R CrA molecular cloud that lie to the east are still embedded in molecular material and appear quite young. It is interesting to note that the intermediate age post-T Tauri stars discovered by Neuhäuser et al. (2000) are spatially located between the main R CrA cloud and the Upper Centaurus Lupus/ Lower Centaurus Crux subgroups in the Sco-Cen OB association. Mamajek \& Feigelson (2001) speculate that R CrA could be a swept up portion of the local ISM formed by the combined affects of supernovae and OB star winds in the last 10-20 Myr. This is consistent with the interpretation that the $\mathrm{R} \mathrm{CrA} \mathrm{cluster}$ is a precursor of unbound small stellar aggregates such as the TW Hya and $\eta$ Cha associations. Perhaps all of these small stellar groups had their origin in the violent activity of the Sco-Cen OB associations.

\section{SUMMARY}

1. We have conducted a near-infrared spectroscopic survey of the embedded cluster associated with the R CrA molecular cloud core. Five objects display emission-line properties suggesting accretion or mass loss. We were able to derive spectral types for 20 objects, five of which are background field stars.

2. We have constructed an H-R diagram for 15 embedded objects located in the cloud core. Ages range from 0.3-3 Myr and masses range from $0.1-2.5 M_{\odot}$. Given the observational 
errors, the age spread in the cloud is consistent with $\delta \tau<$ $3 \mathrm{Myr}$, consistent with the age spreads found in other young clusters (Hartmann 2001; Mamajek et al. 2002).

3 . The properties of the embedded cluster are consistent with other embedded clusters in that the stellar mass function appears to be consistent with having been drawn from the field star IMF (Meyer et al. 2000), and the fraction of objects exhibiting excess emission in the $J H K$ color-color diagram is appropriate for the cluster age (Haisch et al. 2001).

4. The star-forming history of the $\mathrm{R} \mathrm{CrA}$ cloud is suggestive of an origin associated with the Sco-Cen complex. Older post-T Tauri stars are found toward Sco-Cen, while the youngest objects are still embedded in the molecular cloud cores. Perhaps $\mathrm{R}$ CrA was formed through sweeping of the local ISM through the combined action of supernovae and winds from massive stars associated with the Sco-Cen OB association, as suggested by Mamajek and Feigelson (2001).

We would like to dedicate this article to the memory of Richard Elston, who supported the observations presented here, and without whose tireless efforts to improve infrared instrumentation at the National Optical Astronomical Observatory this work would not have been possible.

\section{REFERENCES}

Bessell, M. S. 1991, AJ, 101, 662

Carpenter, J. M., Meyer, M. R., Dougados, C., Strom, S. E., \& Hillenbrand, L. A. 1997, AJ, 114, 1275

Carr, J. S., Tokunaga, A. T., Najita, J., Shu, F. H., \& Glassgold, A. E. 1993, ApJ, 411, L37

Casey, B. W., Mathieu, R. D., Vaz, L. P. R, Andersen, J., \& Suntzeff, N. B. 1998, AJ, 115, 1617

Cohen, J. G., Frogel, J. A., Persson, S. E., \& Elias, J. H. 1981, ApJ, 249,481

Doppmann, G. W., Greene, T. P., Covey, K. R., \& Lada, C. J. 2005, AJ, 130,1145

D’Antona, F., \& Mazzitelli, I. 1994, ApJS, 90, 467

Depoy, D. L., Gregory, B., Elias, J., Montane, A., Perez, G., \& Smith, R. M. 1990, PASP, 102, 1433

de Zeeuw, P. T., Hoogerwerf, R., \& de Bruijne, J. H. J. 1999, AJ, 117,354

Forbrich, J., \& Preibisch, T. 2007, A\&A, 475, 959 (FP07)

Glass, I. S., \& Penston, M. V. 1975, MNRAS, 172, 227

Greene, T. P., \& Lada, C. J. 1996, AJ, 112, 2184

Greene, T. P., \& Meyer, M. R. 1995, ApJ, 450, 233

Haisch, K. E., Lada, E. A., \& Lada, C. J. 2001, ApJ, 553, L153

Hartmann, L. 2001, AJ, 121, 1030

Lada, C. J., \& Lada, E. A. 2003, ARA\&A, 41, 57

Luhman, K. L., et al. 2007, ApJ, 666, 1219

Mamajek, E. E., \& Feigelson, E. D. 2001, in Young Stars Near Earth: Progress and Prospects, ASP Conf. Ser. 244, eds. R. Jayawardhana, \& T. Greene, (San Francisco: ASP), 104

Mamajek, E. E., Meyer, M. R., \& Liebert, J. W. 2002, AJ, 124, 1670

Marraco, H. G., \& Rydgren, A. E. 1981, AJ, 86, 62

Meyer, M. R. 1996, Ph.D. thesis, Univ. Massachusetts

Meyer, M. R., Adams, F. C., Hillenbrand, L. A., Carpenter, J. M., \& Larson, R. B. 2000, in Protostars and Planets IV, eds. V. Mannings,
A. P. Boss, \& S. S. Russell, (Tucson: Arizona Press), 121

Meyer, M. R., Calvet, N., \& Hillenbrand, L. A. 1997, AJ, 114, 288

Meyer, M. R., Edwards, S., Hinkle, K. H., \& Strom, S. E. 1998, ApJ, 508, 397

Neuhäuser, R., \& Forbrich, J. 2008, in Handbook of Star Forming Regions Vol. II, ed. B. Reipurth, (San Francisco: ASP), 735

Neuhäuser, R., Walter, F. M., Covino, E., Alcalá, F., Wolk, S. J., Frink, S., Guillot, P., Sterzik, M. F., \& Comerón, F. 2000, A\&AS, 146, 323

Nisini, B, Antoniucci, S., Giannini, T., \& Lorenzetti, D. 2005, A\&A, 429, 543

Palla, F., \& Stahler, S. W. 2000, ApJ, 540, 255

Patten, B. 1998, in The Tenth Cambridge Workshop on Cool Stars, Stellar Systems and the Sun, ASP Conf. Ser. 154, eds. R. A. Donahue, \& J. A. Bookbinder (San Francisco: ASP), 1755 (P98c)

Schmidt-Kaler, Th. 1982, Landolt-Bornstein New Series, Numerical Data and Functional Relationships in Science and Technology, Group 4, Vol. 2b, ed. K. Schaffers, \& H. H. Voigt, 451

Shu, F. H., Adams, F. C., \& Lizano, S. 1987, ARA\&A, 25, 23

Sicilia-Aguilar, A., Henning, T., Juhá'sz, A., et al. 2008, ApJ, 687,1145

Stahler, S. W. 1988, ApJ, 332, 804

Taylor, K. N. R., \& Storey, J. W. V. 1984, MNRAS, 209, 5 (TS84)

Vrba, F. J., Strom, S. E., \& Strom, K. M. 1976, AJ, 81, 317

Wallace, L., \& Hinkle, K. 1997, ApJS, 111, 445

Wilking, B. A., Greene, T. P., Lada, C. J., Meyer, M. R., \& Young, E. T. 1992, ApJ, 397, 520

Wilking, B. A., Greene, T. P., \& Meyer, M. R. 1999, AJ, 117, 469

Wilking, B. A., McCaughrean, M. J., Burton, M. G., Giblin, T., Rayner, J. T., \& Zinnecker, H. 1997, AJ, 114, 2029 (WMB97)

Wilking, B. A., Meyer, M. R., Greene, T. P., Mikhail, A., \& Carlson, G. 2004, AJ, 127, 1131 